\title{
Gastrectomy: the expanding role of minimally invasive surgery in gastric cancer
}

\author{
Jan Willem van den Berg, Hamish Shilton, Edward Cheong \\ Department of Upper GI Surgery and General Surgery, Norfolk and Norwich University Hospital, Colney lane, Norwich NR4 7UY, \\ United Kingdom.
}

Correspondence to: Prof. Edward Cheong, Department of Upper GI Surgery and General Surgery, Norfolk and Norwich University Hospital, Colney lane, Norwich NR4 7UY, United Kingdom. E-mail: edward.cheong@nnuh.nhs.uk

How to cite this article: van den Berg JW, Shilton H, Cheong E. Gastrectomy: the expanding role of minimally invasive surgery in gastric cancer. Mini-invasive Surg 2019;3:23. http://dx.doi.org/10.20517/2574-1225.2019.07

Received: 27 Feb 2019 First Decision: 11 Jun 2019 Revised: 4 Jul 2019 Accepted: 19 Jul 2019 Published: 30 Jul 2019

Science Editor: Tetsu Fukunaga Copy Editor: Jia-Jia Meng Production Editor: Jing Yu

\begin{abstract}
Gastric cancer remains one of the most frequent cancers worldwide. Currently the only potentially curative treatment is surgery, often in combination with perioperative chemotherapy. Gastric cancer surgery is associated with significant morbidity. However, over the last few decades several potential advances have been introduced to improve the treatment for gastric cancer patients. Introduction of laparoscopic gastric cancer surgery has shown promising results and therefore gained popularity worldwide. This review describes an overview of laparoscopic gastrectomy for gastric cancer patients. In general, the introduction of laparoscopic surgery has shown improvement in the short-term outcomes of gastric cancer treatment. Laparoscopic approach for gastric cancer is feasible, safe and should be performed in experienced high volume centres. However, results from randomised trials in advanced gastric cancer are awaited to further determine the effect of a laparoscopic gastrectomy on oncological and longterm outcomes.
\end{abstract}

Keywords: Gastrectomy, laparoscopy, gastric cancer, laparoscopic gastrectomy, minimal invasive surgery

\section{INTRODUCTION}

Gastric cancer remains the fifth most frequent cancer and the third cause of cancer death worldwide ${ }^{[1]}$. Open gastrectomy $(\mathrm{OG})$ has been the mainstay of curative treatment, however, an open approach is associated with significant morbidity ${ }^{[2]}$. Therefore, laparoscopic gastrectomy (LG) for gastric cancer

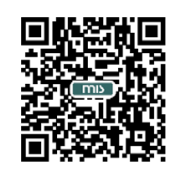


treatment has gained popularity in the last few decades, as it potentially results in a better outcome regarding morbidity and survival. In 1994, a laparoscopic distal gastrectomy (LDG) for early gastric cancer was reported for the first time ${ }^{[3]}$. A few years later, a series of laparoscopic total gastrectomy's (LTG) with D1 and D2 lymph node dissection was published ${ }^{[4]}$. Since then, experience in laparoscopic surgery has improved with time allowing both distal gastrectomy and total gastrectomy to be performed more frequently worldwide. This report outlines the current state of LG for gastric cancer and its future outlook.

\section{LDG FOR GASTRIC CANCER}

Since the report by Kitano et al. ${ }^{[3]}$ in 1994 , LDG has been investigated intensively, predominately in the Far East. A large Korean case control study which compared both LDG and LTG with OG showed no differences in survival, morbidity and mortality ${ }^{[5]}$. Several large randomised controlled trials (RCTs) have been performed, mainly in Asia. The Chinese CLASS-01 trial compared LDG with open distal gastrectomy (ODG) for patients with early stage gastric cancer [T2-4 No-3, Mo (stage 1 gastric cancer) $\left.{ }^{[6]}\right]$. Threeyear disease-free survival was $76.5 \%$ in the laparoscopic group compared with $77.8 \%$ in the open group [1-sided $97.5 \% \mathrm{CI}:(-6.5 \%$ to $\infty)$ ]. In addition, three-year overall survival (OS) rate was $83.1 \%$ in patients who underwent a LDG compared with $85.2 \%$ in the ODG group (HR 1.19; 95\%CI: $0.87-1.64 ; P=0.28$ ). About $40 \%$ of the patients in both treatment groups received adjuvant chemotherapy. The Korean KLASS-01 trial compared LDG with ODG for patients with early stage gastric cancer [T1/2, No/1, Mo (stage 1 gastric cancer $\left.)^{[7]}\right]$. The 5 -year OS was $94.2 \%$ in the laparoscopic group and $93.3 \%$ in the open group $(P=0.64)$. In addition, cancer-specific survival rate was $97.1 \%$ in patients who underwent a LDG compared with $97.2 \%$ in the ODG group $(P=0.91)$. Earlier, the short-term outcomes of the KLASS-01 trial were published ${ }^{[8]}$. Patients who underwent a LDG had a reduced overall complication rate (LDG vs. ODG; $13.0 \%$ vs. $19.9 \%$; $P=0.001$ ), reduced length of stay (LOS) (LDG vs. ODG; $7.1 \pm 3.1$ vs. $7.9 \pm 4.1 ; P<0.001$ ), and a reduced lymph node yield (LDG vs. ODG; $40.5 \pm 15.3$ vs. $43.7 \pm 15.7 ; P<0.001$ ). In addition, post-operative mortality was equal. The Japanese JCOG0912, which compares LDG with ODG, only reported their short term outcomes ${ }^{[9]}$. This study reported that a laparoscopic approach resulted in a longer operation time (LDG vs. ODG; median 278 min vs. $194 \mathrm{~min} ; P<0.001$ ) and reduced blood loss (LDG vs. ODG; median $38 \mathrm{~mL}$ vs. $115 \mathrm{~mL} ; P<$ 0.001). In addition, no differences were reported in mortality and lymph node yield. These studies show that a laparoscopic approach for early stage gastric cancer is safe and reduces the complication rate. However, we will have to wait for the long-term data to interpret the effect of a laparoscopic approach on long-term oncological outcome.

A meta-analysis has compared LDG with ODG including twenty-five studies in the analysis. They reported that a LDG was associated with longer operative times [weighted mean differences (WMD - $48.3 \mathrm{~min} ; P<$ 0.001 )], reduced post-operative complications (OR 0.49; $P=0.002$ ), reduced blood loss (WMD - $118.9 \mathrm{~mL} ; P$ $<0.001$ ), as well as reduced LOS (WMD - 3.6 days; $P<0.001)^{[10]}$. However, patients in the open group had a significant higher lymph node yield (WMD 3.9; $P<0.001$ ). The impact of this reduced lymph node yield on oncological and long-term outcome is unknown. The longer operating time for LDG may be because some surgeons are still on the learning curve. Overall these studies demonstrate that short-term outcomes of LDG for patients with gastric cancer are comparable to ODG.

\section{LTG FOR GASTRIC CANCER}

The laparoscopic approach for LTG has also gained in popularity worldwide since the first series was reported in $1999^{[4]}$. A meta-analysis reviewed the short-term outcomes of LTG compared with OTG in patients with gastric cancer ${ }^{[11]}$. In eight selected studies, LTG was associated with a significant reduction of intraoperative blood loss (WMD - 227.6 mL; 95\%CI: 144.3-310.9; $P<0.001$ ), reduced LOS (WMD - 4.0 days; 95\%CI: 1.4-6.5; $P<0.001$ ) and reduced postoperative complications (RR 0.51; 95\%CI: 0.33-0.77). However, a prolonged operation time was seen in patients who underwent a LTG (WMD - $55.5 \mathrm{~min}$; 95\%CI: 24.8-86.2; 
$P<0.001)$. In addition, there was no difference in the in-hospital mortality between both groups. A further meta-analysis for LTG was performed which reviewed 10 retrospective cohort studies and 2 case-controlled studies ${ }^{[12]}$. A laparoscopic approach for gastric cancer resulted in reduced postoperative complications (OR 0.66; $P=0.02$ ), reduced blood loss (WMD - $160.70 \mathrm{~mL} ; P<0.00001$ ), as well as reduced LOS (WMD 2.43 days; $P=0.0002)$. In addition, there was no significant difference in mortality (OR 0.60, $P=0.52$ ), lymph node yield (WMD - 2.30; $P=0.06$ ), radicality or negative resection margin status. However, again it was observed that a minimally invasive approach resulted in a significantly prolonged operation time (WMD $48.06 \mathrm{~min} ; P<0.00001)$.

A case control study which compared laparoscopic with OG (for both distal and total gastrectomy) showed similar results in line with the meta-analysis. A laparoscopic approach resulted in reduced blood loss, reduced LOS, reduced minor complications and a prolonged length of operation time ${ }^{[13]}$. However, interestingly this study also demonstrated that a laparoscopic approach for gastric cancer resulted in a significant increased likelihood of receiving and completing the course of adjuvant therapy. In the wellknown MAGIC trial only $49.5 \%$ of the patients who successfully completed neo-adjuvant chemotherapy and surgery also completed 3 cycles of adjuvant chemotherapy ${ }^{[14]}$. As adjuvant treatment is critically important for patients with (advanced) gastric cancer this may demonstrate an important benefit of a laparoscopic approach. A propensity scored matched analysis compared LG to OG during the introduction of LG in the Netherlands ${ }^{[15]}$. Analyses in 884 patients showed less wound complications ( $2 \% v s .5 \%, P=$ $0.006)$ and less chyle leakage ( $1 \%$ vs. $4 \%, P=0.004)$ in patients who underwent LG. However, there was no difference in overall postoperative morbidity or anastomotic leakage.

Overall these studies show that LTG is feasible and safe. Further research in the form of RCTs are warranted to confirm short term morbidity and quality of resection, but in particular provide the best data to evaluate the long-term oncological outcomes. So far, most of the studies are conducted in the Far East. A RCT which compares LTG gastrectomy with OTG is currently ongoing ${ }^{[16]}$. The primary endpoint of this study is to evaluate the effect of surgical approach on morbidity and mortality in patients with T1/2, No/1, Mo (stage 1) gastric cancer. More recently, a Korean RCT comparing open with LTG for advanced gastric cancer has started (KLASS-06). However, results from the Far East cannot be easily translated to the Western population. This is mainly due to the different population and body mass index, a less advanced stage of gastric cancer in Asia, and a much lower utilization of (neo)-adjuvant chemotherapy. In Europe two RCTs are ongoing, the STOMACH trial and the LOGICA trial ${ }^{[17,18]}$. Both multicentre randomised trials study the effect of LG with OG in patients with more advanced gastric cancer (T1-3, No-1, Mo) in the Western population. Patients included in these studies predominantly received neoadjuvant chemotherapy. Primary endpoints in these trials are the quality of the oncologic resection and postoperative complications. Recently, preliminary results of these two trials were presented at the International Gastric Cancer Congress 2019. Laparoscopic and OG resulted in comparable oncological outcomes, and comparable morbidity and mortality. Overall, final results of these studies will provide more information on which surgical technique is optimal for treatment of resectable gastric cancer.

\section{COMPLICATIONS FOLLOWING LG}

Gastric cancer surgery is associated with high morbidity. One of the benefits of a laparoscopic approach is reducing morbidity. A propensity matched analysis assessed complications in 4124 patients who underwent a LG or OG, of whom 627 patients developed complications ${ }^{[19]}$. No significant differences were found in overall complications $(14.2 \%$ vs. $16.5 \% ; P=0.093)$. However, hospital mortality was reduced in the LG group $(0.3 \%$ vs. $1.2 \% ; P=0.004)$ as was failure to rescue rates $(2.1 \% v s .7 .6 \% ; P=0.008)$. Multivariate analysis showed that older age, tumour location, TNM stage, extent of gastric resection, operative time and operative blood loss were adverse risk factors for complications. They concluded that whilst overall 
complications were similar, patients were more likely to die when they experienced complications following OG.

A systematic review of 16 non-RCTs of anastomotic complications following LTG vs. OTG did not find any statistical difference in the incidence of anastomotic leakage, 3.0\% vs. 2.1\% respectively (OR 1.42, 95\%CI: 0.86-2.33; $P=0.17)^{[20]}$. The incidence of anastomotic stenosis was also not significantly different between groups, $3.2 \%$ vs. $2.7 \%$ following LTG and OTG respectively (OR 1.55, 95\%CI: 0.94-2.54; $P=0.08$ ). When the LTG was classified into six categories for the various anastomotic techniques, review of the case studies demonstrated a similar anastomotic leak rate (1.1\%-3.2\%), however the incidence of stenosis was relatively high when the $\mathrm{OrVil}^{\mathrm{TM}}$ device was used (8.8\%) compared with other procedures (1.0\%-3.6\%).

\section{LONG TERM ONCOLOGICAL OUTCOMES AND MORBIDITY}

So far, there are no publications of randomised control trials comparing LG with OG which report long term survival data. However, long term survival has been assessed in a meta-analysis looking at 5-year results, for OS, recurrence or gastric cancer related death ${ }^{[21]}$. In this analysis, 23 studies with a total of 7336 patients who underwent a distal or total gastrectomy were included. Excluding the studies that didn't have well balanced groups, they reported no differences between LG and OG as 5-year OS (OR 1.07, 95\%CI: 0.901.28; $P=0.45)$, recurrence (OR 0.83, 95\%CI: 0.68-1.02; $P=0.08)$, and gastric cancer-related death (OR 0.86 , $95 \%$ CI: $0.65-1.13 ; P=0.28)$ rates were similar. They conclude that long term results of LG are comparable to open surgery for both early and advanced gastric cancer.

Large propensity matched cohort studies have been performed to assess the long-term outcomes between OG and LG for advanced gastric cancer to achieve an immediate assessment whilst we await the results of RCTs. Li et al ${ }^{[22]}$ matched 459 and 856 patients undergoing laparoscopic or OG (both distal and total) respectively for advanced gastric cancer with $\mathrm{D} 2$ lymph node resection. No significant difference was identified in the 5 -year OS $(52.0 \%$ and $53.4 \% ; P=0.805)$ and disease-free survival $(46.8 \%$ vs. $47.3 \% ; P=$ 0.963 ) between the laparoscopic and open groups. Stratified assessment also did not identify any differences according to tumour stage. The operation method was not an independent prognostic factor for OS or disease-free survival. In addition, the recurrence pattern was similar between the laparoscopic and OG groups.

Another propensity matched cohort study, the LOC-A study, matched 610 cases with advanced gastric cancer (stage 2/3) who underwent a laparoscopic or open distal, proximal or total gastrectomy ${ }^{[23]}$. No significant differences in 5-year survival or recurrence was found. Five-year survival for OG was 53.0\% compared to $54.2 \%$ following LG. In addition, the recurrence rate was $30.8 \%$ and $29.8 \%$ respectively. High risk patients have also been assessed in a further propensity matched cohort study comparing LG with $\mathrm{OG}^{[24]}$. The patients underwent a distal or total gastrectomy. High risk patients were deemed to have at least one of: age $>80$ years, BMI $>30 \mathrm{~kg} / \mathrm{m}^{2}$, ASA grade $\geq 3$, or clinical T stage 4 . After matching, each group had 341 patients with no difference in clinico-pathological data between the open and laparoscopic groups. Operating time $(181.70 \mathrm{~min} v s .266 .71 \mathrm{~min} ; P<0.001)$ and blood loss $(68.11 \mathrm{~mL} v$ s. $225.54 \mathrm{~mL} ; P<$ 0.001 ) were significantly lower in the LG group, whilst postoperative complications occurred in $11.4 \%$ and $18.5 \%$, in the LG and OG group respectively $(P=0.010)$. Therefore, laparoscopic surgery was a significant protective factor against post-operative complications $(P=0.019)$. In addition, the number of risk factors present was an independent risk factor for post-operative complications $(P=0.021)$. Again, the 5-year OS rate was similar between the LG and OG groups (55.0\% vs. 52.0\%; $P=0.086)$.

\section{ENHANCED RECOVERY AFTER SURGERY}

Enhanced recovery after surgery (ERAS) program has been described for the first time in the nineties ${ }^{[25]}$. Since then, the success of programs has been described in various fields of surgery, including both open 
and laparoscopic techniques in the specialty of Gastro-Oesophageal surgery ${ }^{[26,27]}$. A recent meta-analysis described the results of ERAS in LG patients ${ }^{[28]}$. In this analysis of six studies, with predominantly patients who underwent a LDG, ERAS resulted in shorter LOS (WMD - 2.65; $P<0.01$ ) and less hospitalisation expenditure (WMD - 523.43; $P<0.01$ ). However, no significant difference was found regarding complication rate. This meta-analysis shows that ERAS can be applied to laparoscopic gastric surgery. However, large sample RCT's should be conducted to fully show the effect of an ERAS program in LG patients.

\section{LEARNING CURVE}

A LTG remains a challenging surgical procedure with substantial technical difficulties, such as the extended lymph node dissection and the anastomoses of the oesophagus to the jejunum. Limited studies have addressed the learning curve for LTG. A Korean study described that the learning curve for LTG is approximately 100 cases $^{[29]}$. Earlier, another Korean study described using the cumulative sum technique showed that postoperative morbidity reached a plateau after around $45 \operatorname{cases}^{[30]}$. In addition, for LDG a learning curve of 20 to 40 cases has been reported ${ }^{[31]}$. Overall, these studies show that a lengthy learning curve will be required to achieve acceptable morbidity. In addition, the learning curve for LTG in the Western countries may differ as in general this includes patients with more advanced gastric cancer as well as higher BMI.

\section{CENTRALISATION}

Worldwide centralisation for the care for gastric cancer patients is increasing. In the Netherlands it has been imposed since 2012. A recent analysis on the outcomes from this process has shown an increase in the utilisation of laparoscopic surgery for gastric cancer from $6 \%$ to $40 \%(P<0.01)^{[32]}$. Whilst the volume of laparoscopic surgery has increased, adequate lymphadenectomy has improved from $21 \%$ to $93 \%$ ( $P<$ 0.01 ). Other benefits included decreased median LOS ( 8 days vs. 10 days; $P<0.01$ ), and greater utilisation of perioperative chemotherapy (25\% vs. $42 \% ; P<0.01)$. They reported no significant change in 30 -day mortality $(4.2 \%$ vs. $1.9 \% ; P=0.17), 1$ year overall $(78 \%$ vs. $80 \% ; P=0.17)$ and disease-free survival $(73 \%$ vs. $74 \% ; P=0.66)$. This demonstrates that in western countries, centralisation of gastric cancer results in better rates of laparoscopic resection, improved short term outcomes without impacting on postoperative mortality and intermediate-term survival.

\section{TECHNICAL ASPECTS OF LG}

\section{Anastomoses technique}

LTG is technically difficult. The main concerns are the anastomoses technique and oncological safety. Currently there is no standardised method for the esophago-jejunostomy, and many different techniques have been described. A retrospective study in 687 patients who underwent a LTG compared oesophagojejunostomy using a linear stapler with a circular stapler ${ }^{[33]}$. Complication rates were similar between the two groups, however the linear stapler resulted in a shorter operation time (149 min vs. $170 \mathrm{~min}, P<$ 0.001 ) as well as shorter length of hospital stay. Therefore, concluded was that a linear stapler technique for an oesophago-jejunostomy is a feasible procedure. A retrospective study compared a circular stapler $\left(\mathrm{OrVil}{ }^{\mathrm{TM}}\right)$ esophago-jejunostomy with an overlap esophago-jejunostomy using a linear stapler ${ }^{[34]}$. The rate of anastomotic anastomotic leakage was lower in the linear stapler group compared with the OrVil ${ }^{\mathrm{TM}}$ group ( $0.7 \% v$ s. $4.1 \%)$ although this was not significant. In addition, the rate of anastomotic stenosis was significantly lower in the linear stapler group compared with the $\mathrm{OrVil}^{\mathrm{TM}}$ group $(0.0 \%$ vs. $4.1 \%, P=0.017)$. However, there are no RCT's comparing the optimal anastomoses technique in patients undergoing LTG.

\section{Extent of lymph node dissection}

Numerous studies have reported the impact of lymph node dissection on survival in gastric cancer surgery. Fifteen year follow up of a Dutch trial comparing a limited lymph node dissection (D1) with an extended 
lymph node dissection (D2) reported that D2 lymphadenectomy is associated with lower locoregional recurrence $(\mathrm{D} 1 ; 22 \% v s . \mathrm{D} 2 ; 12 \%)$ and significantly reduced gastric-cancer related deaths $(\mathrm{D} 1 ; 48 \% v s$. D2; 37\% ${ }^{[35]}$. A systematic review of RCTs comparing different types of lymph node dissection in patients with gastric cancer was reported ${ }^{[36]}$. In this review no significant difference in OS was seen after D1 lymphadenectomy compared with D2 lymphadenectomy ( $n=5$; HR 0.91, 95\%CI: 0.71 to 1.17). In contrast, D2 lymphadenectomy was related to a significantly improved disease specific survival compared to D1 lymphadenectomy (HR 0.81, 95\%CI: 0.71-0.92). However, the D2 lymphadenectomy was associated with a higher postoperative mortality rate (RR 2.02, 95\%CI: 1.34-3.04). The high post-operative morbidity and mortality following D2 dissection in this study was attributed to performing a splenectomy. Therefore, a spleen-preserving D2 lymphadenectomy is the recommend surgical approach for resectable advanced gastric cancer.

In a meta-analysis comparing open with LG, 11 of 12 studies described lymph node resection, with no significant differences between OTG and LTG groups ${ }^{[12]}$. Eight of the studies individually favoured open resection for lymph node resection. Only three studies reported resection margin status, with no difference in proximal, distal or circumferential margin Ro resection status. Overall, lymph node yield was equal after both methods of gastric surgery. The recently published long-term results of the CLASS-01 trial showed a mean retrieval of lymph nodes of 36 in the laparoscopic group compared with 37 in the open group ${ }^{[6]}$. Overall, these numbers are higher compared with the number of lymph nodes retrieved in the West. Therefore, the results of ongoing RCTs in Europe are needed to establish the optimal surgical approach in patients with gastric cancer in Western countries.

\section{Omentectomy}

A Dutch prospective trial studied the presence of metastases in the greater omentum in patients undergoing a (sub) total gastrectomy with omentectomy and D2 lymphadenectomy for gastric cancer ${ }^{[37]}$. Five percent of the patients had metastases in the greater omentum, however all these patients also had a positive proximal or distal resection margin (R1 resection). In addition, another Dutch prospective trial demonstrated that omental lymph node metastases or tumour deposits are present in $10 \%$ of the patients ${ }^{[38]}$. Worldwide there is no consensus on whether to perform an omentectomy or not for gastric cancer surgery. RCTs are warranted to investigate the effect of omentectomy on long-term survival. Traditionally, an omentectomy has been considered the standard approach to gastrectomy for gastric cancer patients. Due to possible presence of omental lymph node metastases and difficulty in predicting presence of these nodes, omentectomy should be performed as standard, unless proven otherwise by randomised trials.

\section{Bursectomy}

In eastern Asia, a bursectomy has been performed to remove the peritoneum covering the pancreas and the anterior plane of the transverse mesocolon. Two Japanese RCTs compared bursectomy with omentectomy for patients with resectable gastric cancer. First, Hirao et al ${ }^{[39]}$ reported in patients with cT2-3 gastric adenocarcinoma and D2 gastrectomy a 5-year survival of $77.5 \%$ in patients who underwent a bursectomy compared with $71.3 \%$ in patients without bursectomy $(P=0.16)$. However, multivariate analysis that bursectomy was an independent prognostic factor of good OS $(P=0.033)$. More recently, Kurokawa et al. ${ }^{[4]}$ reported no survival advantage for bursectomy combined with omentectomy over omentectomy alone. Five-year OS was 76.7\% in the non-bursectomy group and 76.9\% in the bursectomy group (HR 1.05, 95\%CI: 0.81-1.37; $P=0.65$ ). In addition, a recent meta-analysis reported that a bursectomy for advanced gastric cancer has no positive influence on the number of harvested lymph nodes (WMD 5.86; $P=0.157$ ) or on the OS (HR 0.95; $P=0.647)^{[41]}$. A study describing patients who underwent a LTG with complete bursectomy showed that this technique is feasible and safe in experienced hands ${ }^{[42]}$. 


\section{Jejunal pouch}

The most common reconstruction technique after a total gastrectomy is the oesophago-jejunostomy Rouxen-Y reconstruction. However, this technique is associated with complaints like reflux, weight loss, and dumping syndrome ${ }^{[43]}$. One of the techniques performed to potentially improve long-term outcome is the formation of a jejunal pouch ${ }^{[4]}$. A recent meta-analysis which includes 25 studies comparing reconstruction with or without a pouch, mainly in patients who underwent open surgery, showed a reduction of the risk of dumping syndrome $e^{[45]}$, reduced heartburn and oesophagitis, as well as a significantly higher body mass index (22.2 kg/m² vs. $20.9 \mathrm{~kg} / \mathrm{m}^{2}$; WMD 1.28; 95\%CI: 0.61-1.94). However, minimal reports of jejunal pouch reconstruction in a laparoscopic approach have been published so far ${ }^{[46]}$.

\section{NOVEL TECHNIQUES}

\section{Robotic gastrectomy}

Robotic surgery for gastric cancer remains experimental and controversial, in part due to the lack of RCTs. However, several Western and Asian centres have reported their series of robotic surgery in gastric cancer patients. A meta-analysis comparing LG to robotic gastrectomy included three non-randomised controlled studies ${ }^{[47]}$. Robotic gastrectomy was associated with a longer operation time but reduced intra-operative blood loss. No differences were found in lymph node yield, morbidity, mortality, and LOS. The authors therefore concluded that a robotic approach is safe and feasible, however, that further research is warranted to investigate the effect on long-term oncological outcomes. More recently, a meta-analysis which included 19 studies comparing LG with robotic gastrectomy again showed that robotic gastrectomy was associated with a prolonged operation time (WMD - $49.05 \mathrm{~min} ; P<0.01)^{[48]}$. In addition, reduced intraoperative blood loss (WMD - $24.38 \mathrm{~mL} ; P<0.01$ ) as well as higher costs were observed (WMD - 3944.8 USD; $P<0.01$ ). Again, there were no differences in morbidity, mortality, LOS, and lymph node yield. In general, robotic gastrectomy is as safe as LG. However, as the robotic approach is associated with longer operation times and higher costs, without any significant advantages over LG, the laparoscopic approach for gastric cancer is generally preferred.

\section{CONCLUSION}

Minimally invasive gastrectomy is feasible and safe in eastern and western countries. It has benefits for both early and advanced gastric cancer, as well as total and subtotal gastrectomy. The increasing worldwide trend for centralisation of gastric cancer treatment facilitates the use of a laparoscopic approach. These both aspects will increase treatment with neo-adjuvant and adjuvant chemotherapy, whilst LG also improves tolerance of adjuvant chemotherapy. Short term benefits include less blood loss, decreased LOS and lower postoperative morbidity and mortality. Quality of surgery markers such as lymph node yield and resection margin status appear to be comparable to open surgery. Long term survival data available currently indicate overall and disease-free survival is not inferior to open surgery, however the results from ongoing RCTs are awaited.

\section{DECLARATIONS}

\section{Authors' contributions}

All authors had equal contribution in the writing process of this article.

\section{Availability of data and materials}

Not applicable.

\section{Financial support and sponsorship}

None. 


\section{Conflicts of interest}

All authors declared that there are no conflicts of interest.

\section{Ethical approval and consent to participate}

Not applicable.

\section{Consent for publication}

Not applicable.

\section{Copyright}

(c) The Author(s) 2019.

\section{REFERENCES}

1. Ferlay J, Soerjomataram I, Dikshit R, Eser S, Mathers C, et al. Cancer incidence and mortality worldwide: sources, methods and major patterns in GLOBOCAN 2012. Int J Cancer 2015;136:E359-86.

2. Memon MA, Subramanya MS, Khan S, Hossain MB, Osland E,et al. Meta-analysis of D1 versus D2 gastrectomy for gastric adenocarcinoma. Ann Surg 2011;253:900-11.

3. Kitano S, Iso Y, Moriyama M, Sugimachi K. Laparoscopy-assisted Billroth I gastrectomy. Surg Laparosc Endosc 1994;4:146-8.

4. Azagra JS, Goergen M, De Simone P, Ibanez-Aguirre J. Minimally invasive surgery for gastric cancer. Surg Endosc 1999;13:351-7.

5. Kim HH, Han SU, Kim MC, Hyung WJ, Kim W, et al. Long-term results of laparoscopic gastrectomy for gastric cancer: a large-scale case-control and case-matched Korean multicenter study. J Clin Oncol 2014;32:627-33.

6. Yu J, Huang C, Sun Y, Su X, Cao H, et al. Effect of Laparoscopic vs Open Distal Gastrectomy on 3-Year Disease-Free Survival in Patients With Locally Advanced Gastric Cancer: The CLASS-01 Randomized Clinical Trial. Jama 2019;321:1983-92.

7. Kim HH, Han SU, Kim MC, Kim W, Lee HJ, et al. Effect of Laparoscopic Distal Gastrectomy vs Open Distal Gastrectomy on Longterm Survival Among Patients With Stage 1 Gastric Cancer: The KLASS-01 Randomized Clinical Trial. JAMA Oncol 2019;5:505513.

8. Kim W, Kim HH, Han SU, Kim MC, Hyung WJ, et al. Decreased Morbidity of Laparoscopic Distal Gastrectomy Compared With Open Distal Gastrectomy for Stage I Gastric Cancer: Short-term Outcomes From a Multicenter Randomized Controlled Trial (KLASS-01). Ann Surg 2016;263:28-35.

9. Katai H, Mizusawa J, Katayama H, Takagi M, Yoshikawa T, et al. Short-term surgical outcomes from a phase III study of laparoscopyassisted versus open distal gastrectomy with nodal dissection for clinical stage IA/IB gastric cancer: Japan Clinical Oncology Group Study JCOG0912. Gastric Cancer 2017;20:699-708.

10. Vinuela EF, Gonen M, Brennan MF, Coit DG, Strong VE. Laparoscopic versus open distal gastrectomy for gastric cancer: a metaanalysis of randomized controlled trials and high-quality nonrandomized studies. Ann Surg 2012;255:446-56.

11. Haverkamp L, Weijs TJ, van der Sluis PC, van der Tweel I, Ruurda JP, et al. Laparoscopic total gastrectomy versus open total gastrectomy for cancer: a systematic review and meta-analysis. Surg Endosc 2013;27:1509-20.

12. Straatman J, van der Wielen N, Cuesta MA, de Lange-de Klerk ES, Jansma EP, et al. Minimally Invasive Versus Open Total Gastrectomy for Gastric Cancer: A Systematic Review and Meta-analysis of Short-Term Outcomes and Completeness of Resection : Surgical Techniques in Gastric Cancer. World J Surg 2016;40:148-57.

13. Kelly KJ, Selby L, Chou JF, Dukleska K, Capanu M, et al. Laparoscopic Versus Open Gastrectomy for Gastric Adenocarcinoma in the West: A Case-Control Study. Ann Surg Oncol 2015;22:3590-6.

14. Cunningham D, Allum WH, Stenning SP, Thompson JN, Van de Velde CJ, et al. Perioperative chemotherapy versus surgery alone for resectable gastroesophageal cancer. N Engl J Med 2006;355:11-20.

15. Brenkman HJF, Gisbertz SS, Slaman AE, Goense L, Ruurda JP, et al. Postoperative Outcomes of Minimally Invasive Gastrectomy Versus Open Gastrectomy During the Early Introduction of Minimally Invasive Gastrectomy in the Netherlands: A Population-based Cohort Study. Ann Surg 2017;266:831-8.

16. He H, Li H, Su X, Li Z, Yu P, et al. Study on safety of laparoscopic total gastrectomy for clinical stage I gastric cancer: the protocol of the CLASS02-01 multicenter randomized controlled clinical trial. BMC Cancer 2018;18:944.

17. Haverkamp L, Brenkman HJ, Seesing MF, Gisbertz SS, van Berge Henegouwen MI, et al. Laparoscopic versus open gastrectomy for gastric cancer, a multicenter prospectively randomized controlled trial (LOGICA-trial). BMC Cancer 2015;15:556.

18. Straatman J, van der Wielen N, Cuesta MA, Gisbertz SS, Hartemink KJ, et al. Surgical techniques, open versus minimally invasive gastrectomy after chemotherapy (STOMACH trial): study protocol for a randomized controlled trial. Trials 2015;16:123.

19. Tu RH, Lin JX, Zheng CH, Li P, Xie JW, et al. Complications and failure to rescue following laparoscopic or open gastrectomy for gastric cancer: a propensity-matched analysis. Surg Endosc 2017;31:2325-37.

20. Inokuchi M, Otsuki S, Fujimori Y, Sato Y, Nakagawa M, et al. Systematic review of anastomotic complications of esophagojejunostomy after laparoscopic total gastrectomy. World J Gastroenterol 2015;21:9656-65.

21. Chen XZ, Wen L, Rui YY, Liu CX, Zhao QC, et al. Long-term survival outcomes of laparoscopic versus open gastrectomy for gastric 
cancer: a systematic review and meta-analysis. Medicine (Baltimore) 2015;94:e454.

22. Li Z, Li B, Bai B, Yu P, Lian B, et al. Long-term outcomes of laparoscopic versus open D2 gastrectomy for advanced gastric cancer. Surg Oncol 2018;27:441-8.

23. Kinoshita T, Uyama I, Terashima M, Noshiro H, Nagai E, et al. Long-term Outcomes of Laparoscopic Versus Open Surgery for Clinical Stage II/III Gastric Cancer: A Multicenter Cohort Study in Japan (LOC-A Study). Ann Surg 2019;269:887-94.

24. Xu BB, Lu J, Zheng ZF, Huang CM, Zheng CH, et al. Comparison of short-term and long-term efficacy of laparoscopic and open gastrectomy in high-risk patients with gastric cancer: a propensity score-matching analysis. Surg Endosc 2019;33:58-70.

25. Bardram L, Funch-Jensen P, Jensen P, Crawford ME, Kehlet H. Recovery after laparoscopic colonic surgery with epidural analgesia, and early oral nutrition and mobilisation. Lancet 1995;345:763-4.

26. Preston SR, Markar SR, Baker CR, Soon Y, Singh S, et al. Impact of a multidisciplinary standardized clinical pathway on perioperative outcomes in patients with oesophageal cancer. Br J Surg 2013;100:105-12.

27. Kang SH, Lee Y, Min SH, Park YS, Ahn SH, et al. Multimodal Enhanced Recovery After Surgery (ERAS) Program is the Optimal Perioperative Care in Patients Undergoing Totally Laparoscopic Distal Gastrectomy for Gastric Cancer: A Prospective, Randomized, Clinical Trial. Ann Surg Oncol 2018;25:3231-8.

28. Li MZ, Wu WH, Li L, Zhou XF, Zhu HL, et al. Is ERAS effective and safe in laparoscopic gastrectomy for gastric carcinoma? A meta-analysis. World J Surg Oncol 2018;16:17.

29. Jung DH, Son SY, Park YS, Shin DJ, Ahn HS, et al. The learning curve associated with laparoscopic total gastrectomy. Gastric Cancer 2016;19:264-72.

30. Jeong O, Ryu SY, Choi WY, Piao Z, Park YK. Risk factors and learning curve associated with postoperative morbidity of laparoscopic total gastrectomy for gastric carcinoma. Ann Surg Oncol 2014;21:2994-3001.

31. Kim HG, Park JH, Jeong SH, Lee YJ, Ha WS, et al. Totally laparoscopic distal gastrectomy after learning curve completion: comparison with laparoscopy-assisted distal gastrectomy. J Gastric Cancer 2013;13:26-33.

32. Nelen SD, Heuthorst L, Verhoeven RHA, Polat F, Kruyt PM, et al. Impact of Centralizing Gastric Cancer Surgery on Treatment, Morbidity, and Mortality.J Gastrointest Surg 2017;21:2000-8.

33. Gong CS, Kim BS, Kim HS. Comparison of totally laparoscopic total gastrectomy using an endoscopic linear stapler with laparoscopic-assisted total gastrectomy using a circular stapler in patients with gastric cancer: A single-center experience. World J Gastroenterol 2017;23:8553-61

34. Kawamura H, Ohno Y, Ichikawa N, Yoshida T, Homma S, et al. Anastomotic complications after laparoscopic total gastrectomy with esophagojejunostomy constructed by circular stapler (OrVil()) versus linear stapler (overlap method). Surg Endosc 2017;31:5175-82.

35. Songun I, Putter H, Kranenbarg EM, Sasako M, van de Velde CJ. Surgical treatment of gastric cancer: 15-year follow-up results of the randomised nationwide Dutch D1D2 trial. Lancet Oncol 2010;11:439-49.

36. Mocellin S, McCulloch P, Kazi H, Gama-Rodrigues JJ, Yuan Y, et al. Extent of lymph node dissection for adenocarcinoma of the stomach. Cochrane Database Syst Rev 2015:CD001964.

37. Jongerius EJ, Boerma D, Seldenrijk KA, Meijer SL, Scheepers JJ, et al. Role of omentectomy as part of radical surgery for gastric cancer. Br J Surg 2016;103:1497-503.

38. Haverkamp L, Brenkman HJ, Ruurda JP, Ten Kate FJ, van Hillegersberg R. The Oncological Value of Omentectomy in Gastrectomy for Cancer. J Gastrointest Surg 2016;20:885-90

39. Hirao M, Kurokawa Y, Fujita J, Imamura H, Fujiwara Y, et al. Long-term outcomes after prophylactic bursectomy in patients with resectable gastric cancer: Final analysis of a multicenter randomized controlled trial. Surgery 2015;157:1099-105.

40. Kurokawa Y, Doki Y, Mizusawa J, Terashima M, Katai H, et al. Bursectomy versus omentectomy alone for resectable gastric cancer (JCOG1001): a phase 3, open-label, randomised controlled trial. Lancet Gastroenterol Hepatol 2018;3:460-8.

41. Nie RC, Yuan SQ, Chen S, Yan SM, Chen YM, et al. Bursectomy for advanced gastric cancer: an update meta-analysis. World J Surg Oncol 2018;16:66.

42. Zou L, Xiong W, Mo D, Chen G, He Y, et al. Totally laparoscopic complete bursectomy and D2 lymphadenectomy in radical total gastrectomy: an outside bursa omentalis approach. Surg Endosc 2016;30:4152.

43. Liedman B. Symptoms after total gastrectomy on food intake, body composition, bone metabolism, and quality of life in gastric cancer patients--is reconstruction with a reservoir worthwhile? Nutrition 1999;15:677-82.

44. Lehnert T, Buhl K. Techniques of reconstruction after total gastrectomy for cancer. Br J Surg 2004;91:528-39.

45. Syn NL, Wee I, Shabbir A, Kim G, So JB. Pouch Versus No Pouch Following Total gastrectomy: Meta-analysis of Randomized and Non-randomized Studies. Ann Surg 2019;269:1041-53.

46. Brenkman HJ, Correa-Cote J, Ruurda JP, van Hillegersberg R. A Step-Wise Approach to Total Laparoscopic Gastrectomy with Jejunal Pouch Reconstruction: How and Why We Do It. J Gastrointest Surg 2016;20:1908-15.

47. Xiong B, Ma L, Zhang C. Robotic versus laparoscopic gastrectomy for gastric cancer: a meta-analysis of short outcomes. Surg Oncol 2012;21:274-80.

48. Chen K, Pan Y, Zhang B, Maher H, Wang XF, et al. Robotic versus laparoscopic Gastrectomy for gastric cancer: a systematic review and updated meta-analysis. BMC Surg 2017;17:93. 